(Aus dem pharmakologischen Institut der Universität Breslau.)

\title{
Chemische und thermische Reizung am strychninisirten Frosche.
}

Von

Hans Eekhardt, approb. Arzt.

Mit der Wirkung der verschiedenen Reizarten auf strychninbehandelte Frösche hat sich (1890) eine Arbeit von Schlick aus dem physiologischen Institut zu Jena beschäftigt: „Zur Kenntniss der Strychninwirkung", dieses Archiv, Bd. 47, S. 171. Dass die Reaction auf tactilen und faradischen Reiz im Stadium der Vergiftung verstärkt ist, betrachtet Schlick mit Recht als erwiesen. Er beschäftigt sich speciell mit dem chemischen Reize und kommt auf Grund seiner Versuche zu dem Schlusse, dass für diesen eine bedleutende Verminderung der Erregbarkeit beim Strychninfrosche vorbanden sei; der thermische Reiz dagegen soll nach seiner Angabe (1. c. S. 186) dieselben Erscheinungen hervorrufen wie der tactile und der faradische.

Diese Angabe muss um so mehr in Erstaunen versetzen, als man hätte erwarten mögen, dass sich einerseits allerdings der faradische cutane Reiz mehr ähnlich dem tactilen, andererseits aber der thermische mehr ähnlich dem chemischen Reize verhalten würde. Uebrigens ist die Behauptung in Betreff des thermischen Reizes kaum durch Schlick's thatsächliche Angaben recht gestützt. Denn es findet sich darüber bei ihm nur die kurze Notiz, dass "durch Eintauchen der Zehen in Wasser von $50^{\circ}$ beim unvergifteten wie beim vergifteten Thiere mit Sicherheit Reflexbewegungen ausgelöst wurden" (1. c. S. 186). Eine Steigerung der Erregbarkeit nach der Vergiftung wird also nicht erwähnt; Versuchsprotokolle sind nicht angeführt.

Ist somit die Behauptung einer Gegensätzlichkeit zwischen Wirkung des thermischen und des chemischen Reizes eigentlich unberechtigt, so zeigt eine schärfere Ueberlegung noch ausserdem, dass auch Schlick's Behauptung bezüglich der durch Strychnin bedingten Abschwächung der Empfindlichkeit gegen den chemischen 
Reiz vielleicht schon eine nicht ausreichende Versuchsanordnung zur Seite hat. Bei Schliek wird der enthirnte Frosch mittelst eines durch den Oberkiefer gezogenen Farlens frei aufgehängt, und nunmehr werden die herabhängenden Beine abwechselnd gereizt (durch Eintauchen der längsten Zehe in die reizende Flüssigkeit).

Es ist natürlich zuzugeben, dass sonst Aenderungen der allgemeinen Reflexerregbarkeit, oder richtiger gesagt, der allgemeinen Empfindlichkeit, Erhöhung und Abschwächung, auf diese Weise wohl festgestellt werden können, auch chemischen Reizen gegenüber; aber es muss sich eben um die Frage nach der allgemeinen Sensibilität. dabei handeln. Man wird daher gut thun, die Frage, die Schlick in Angriff genommen hat, zu theilen: chemischen Reizen gegenüber festzustellen,

1. wie weit die allgemeine Empfindlichkeit unter Einführung des Strychnins zu- oder abnimmt;

2. wie die specifische, noch zu besprechende Reflexreaction gerade gegen den chemischen Reiz sich etwa geändert hat, und insbesondere, wie weit Irradiation dieser Reflexe und besonders der Uebergang in Streckreflexe, wie sie für Strychnin ja bekanntlich so charakteristisch sind, bei chemischen Reizen festgestellt werden kann.

Auf die erste dieser Fragen geben Schlick's Versuche eine Antwort, da man, wie gesagt, Aenderungen der allgemeinen Empfindlichkeit am hängenden Präparate wohl prüfen kann. Ausserdem hat sich aber beim Frosche eine specifische Reaction gegen den chemischen Reiz ausgebildet, die ihren Grund in dessen eigenartiger Wirkung hat. Mit dem blossen Wegziehen des betroffenen Gliedes ist nämlich offenbar der Reiz noch nicht beseitigt; die anhaftende Flüssigkeit wirkt fort. Daher wischt bekanntlich der Frosch nit der Hinterpfote an der betroffenen Stelle, um das reizende Agens wegzubringen. Diesen Reactionsvorgang zu veranschaulichen, gelingt natürlich nicht am hängenden Präparat; man muss vielmehr den Frosch in hockender Stellung untersuchen, um die specifische Reaction auf den chemischen Reiz studiren zu können.

Es wäre nun sehr wohl möglich, dass bei dieser Untersuchungsart ein anderes Resultat sich ergäbe wie am hängenden Präparat.

Whe ich aber meine hierher gehörigen Versuche bespreche, kehre ich zu der Frage nach der allgemeinen (Reflex-)Empfindlichkeit zurück. Schlick's Versuche, die sämmtlich hierber gehören, gaben, wie schon erwähnt, das Resultat, dass ein ausgesprochener Gegensatz 
bestünde zwischen dem chemischen Reize und den anderen Reizarten. Gegen die Art, wie Schlick diese Untersuchungen durchführt, ist nichts Wesentliches einzuwenden. Entscheidend ist ein am Schlusse der Arbeit angeführter Versuch, bei dem an demselben Frosche nebeneinander faradische und chemische Reize applicirt und die Resultate verglichen werden. Es zeigt sich hier, dass die Empfindlichkeit gegen den faradischen Reiz nach der Vergiftung etwas zunimmt und bei einer grösseren Anzahl auf einander folgender Prüfungen gesteigert bleibt; dagegen nimmt die Empfindlichkeit gegen den chemischen Reiz stetig ab.

Wenn auch dies Resultat unanfechtbar scheint, so gestatten doch die von Schlick dargereichten hohen Strychningaben einen Einwand. Seine grösste Dosis war $2^{1 / 2} \mathrm{mg}$, die kleiuste etwa $2 / \mathrm{s} \mathrm{mg}$. Die günstigsten Strychningaben für solche Untersuchungen sind aber ${ }^{1 / 20}$ bis ${ }^{1 / 10} \mathrm{mg}$; je weiter man diese Mengen übersteigt, desto zeitiger macht sich die lähmende Wirkung des Strychnins geltend.

Ich habe nun eine Anzahl Versuche mit kleinen Dosen angestellt und habe ferner die Versuche Schlick's dahin modificirt, dass ich für jeden einzelnen Frosch diejenige Concentration des chemischen Reizmittels feststellte, die vor der Vergiftung gerade noch eine Reaction hervorrief. Schlick hat es unterlassen, diese Reizschwelle festzustellen. Er wendet Essigsäureverdünnungen von 0,5 bis $1 \%$ an. Nun ist aber 0,5\% \% ige Essigsäure schon ein sehr starker Reiz für die Froschhaut, von dem überdies zu befürchten ist, dass er bei häufiger Anwendung schädigend auf sie einwirkt. Aus diesem Grunde ist also ein Vergleich der Resultate von elektrischer und chemischer Reizung, wie Schlick sie anstellte, nicht zulässig; denn bei dem oben citirten Versuche hat er für den elektrischen Reiz vor der Vergiftung den Schwellenwerth bestimmt, für den chemischen nicht. Dass Schlick durch abwechselnde Anwendung von 0,5- und $1 \%$ iger Essigsäure nun gar "den Unterschied zwischen starken und schwachen Lösungen kennen lernen" konnte, ist ganz ausgeschlossen, da, wie gesagt, schon $0,5 \%$ ige Essigsäure ein sehr starker Reiz ist. Denn nach meinen Erfahrungen zieht der enthirnte Frosch noch aus einer $0.05 \%$ igen Essigsäureverdünnung die Beine heraus.

Schliesslich weisen Schlick's Versuchsprotokolle insofern eine Uivollkommenheit auf, als sie nur die Angabe der Zeitdauer enthalten, bis die Reaction eintrat, nicht aber eine genauere Beschreibung ser Reaction selhst, betreffend die Energie und etwaige Irradiation, 
der Reflexe. Nun ist aber das Messen der zwischen Eintauchen in die reizende Flüssigkeit und Eintreten der Reaction ablaufenden Zeit nicht das geeignete Mittel, um eine Aenderung der Erregbarkeit festzustellen. Denn verkürzt wird durch Strychnin nur die Zeit des Ablaufes der Erregung im centralen Theile des Reflexbogens; hierbei handelt es sich um Bruchtheile einer Secunde, die nur durch die feinsten Instrumente gemessen werden können. Diejenige Zeit, welche zwischen Eintauchen und Reaction vergeht und auch bei Schlick mehrere - 10 bis 12 - Secunden beträgt, ist also gar nicht die Reflexzeit; bei einer richtig gewählten (schwachen) Concentration der Säurelösung wird die Grösse dieser Secundenzahl bestimmt durch die Zeit, welche nothwendig ist, bis die allmälig in die Haut diffundirende Säure an Ort und Stelle stark genug geworden ist, um auf die sensible Nervenendigung als Reiz einzuwirken. Dagegen tritt bei anderen Reizarten die Erregung sofort ein.

Ein anderer Unterschied des chemischen Reizes von dem tactilen und faradischen besteht darin, dass die Erregung andauert und zunimmt bis zur Beseitigung der Süure. Wir haben es also mit allmälig wachsendem und sich central summirendem Reize $\mathrm{zu}$ thun, während die anderen mehr oder weniger plötzlich hereinbrechende, mehr oder weniger momentane Reizungen darstellen.

Es war also meine Aufgabe, zu constatiren,

1. welches die minimale Säureconcentration ist, aus welcher der Frosch die Beine noch herauszieht, vor und nach der Vergiftung; mit anderen Worten: ob die Empfindlichkeit zu- oder abnimmt;

2. $o b$ aus einer sachgemäss gewählten Concentration der Strychninfrosch im Stadium der tactilen Reflexübererregbarkeit wirklich wesentlich später das Bein herauszieht als in der Norm, wie Schlick behauptet. Eine unveränderte Zeitdauer zwischen Eintauchen und Reaction kann nach dem oben Erörterten mit einer sehr wesentlichen Verkürzung der eigentlichen Reflexzeit ganz wohl verbunden sein, und diese kann beim chemischen Reize nach keiner Methode bestimmt werden; wohl aber sind wir in der Lage, durch Bestimmung des Reizschwellenwerthes und der Energie der Reaction einen Maassstab für die Reflexerregbarkeit zu gewinnen.

Bei meinen unter Berücksichtigung der erwähnten Punkte angestellten Versuchen hat sich nun gezeigt, dass diese Zeit zwischen Application des Reizes und Reaction vor und nach der Vergiftung fast dieselbe ist; Schwankungen von einigen Secunden kommen so- 
wohl vorher wie nachher vor. Andererseits gelingt es im Stadium der Reflexübererregbarkeit stets, zu zeigen, dass der Frosch auf einen vorher eben noch nicht wirksamen beziehungsweise eben wirksamen Reiz jetzt schon reagirt beziehungsweise viel intensiver reagirt, so dass hier der vorher nur in Herausziehen bestehende Reflex durch Irradiation zu Allgemeinbewegungen sich steigert, und dass schliesslich auf der Höhe der Vergiftung ein Krampf erfolgt.

Sobald jedoch der erste Krampf stattgefunden hat, sinkt die Anspruchsfähigkeit des Präparates für das vorher ausreichende chemische Reizmittel ab, während eine Berührung, oft sogar das Berühren der Wasseroberfäche, noch prompt einen Krampfanfall auslöst. Erst von diesem Punkte an stimmen also meine Versuche mit denen Schlick's überein, und übrigens auch nur scheinbar.

Diese Verminderung der Empfindlichkeit gegen den chemischen Reiz ist nämlich nicht endgiltig, wie folgende Beobachtung zeigt. Von der Vergiftung mit $1 / 20$ bis $1 / 10 \mathrm{mg}$ Strychnin erholen sich die Präparate meist in kurzer Zeit so weit, dass auf Berührungen nicht wie vorher ein Krampfanfall von mehreren Secunden bis zu 1/2 Minute Dauer erfolgt, sondern nur kurze Krampfstösse, häung mit vorangehendem Versuche, das betroffene Bein anzuziehen. Lässt man in diesem Starlium die Präparate einige Minuten in Ruhe und reizt dann chemisch, so findet man dieselbe Empfindlichkeit wie im Anfangsstadium der Vergiftung, d. h. die Beine werden jetzt nach ungefähr derselben Zeit wie zu Anfang ebenso kräftig herausgezogen; gleich darauf folgt ein kurzer Krampfstoss. -- Die Verminderung der Erregbarkeit gegen den chemischen Reiz ist also nur vorübergehend, und zwar nach dem eben Gesagten zusammenhängend mit der Frschöpfung, welche die Krämpfe hervorbringen. Unzweifelhaft bewirkt diese Erschöpfung eine geringe Abnahme der Empfindlichkeit auch gegen den tactilen Reiz; obschon er immer noch im Vergleich zur Norm verstärkt ist, so ist er doch weniger wirksam als im ersten Stadium der Vergiftung. Hingegen ist, wie gesagt, der vor der Vergiftung noch wirksame schwächste chemische Reiz jetzt unwirksam.

Dieses entgegengesetzte Verhalten des chemischen und (beispielsweise) des tactilen Reizes beruht offenbar auf ihrer schon angedeuteten verschiedenen Wirkungsweise, die hier näher besprochen werden mag. Dem tactilen Reize eigenthümlich ist das plötzliche Hereinbrechen und Wiederverschwinden. Wollte man seine Curve aufzeichnen mit Ordinaten auf einer Abscisse, welche die zwischen Reizung und Re- 
action verlaufende Zeit bedeutet, so müsste man sie als eine fast senkrecht aufsteigende, auf der Höhe in spitzem Winkel umbrechende und fast senkrecht wieder abfallende Linie zeichnen. Der chemische Reiz dagegen ist charakterisirt durch allmäliges Ansteigen seiner Curve; denn im Augenblicke der Application noch unwirksam, dringt die Flüssigkeit nur allmälig in die Haut ein, bis der Höhepunkt erreicht ist. Ebenso fällt der absteigende Schenkel dieser Curve allmälig $a b$; denn beim Abspülen des Reizmittels wird dieses nicht mit einem Schlage, sondern nach und nach von der Nervenendigungr entfernt. Analoges muss für den thermischen Reiz gelten.

Auf Grund dieser Ueberlegung ist die Schlussfolgerung berechtigt, dass ein durch langdauernden Krampf geschwächtes Präparat zwar bei Anwendung des tactilen und faradischen Reizes noch eine deutliche Uebererregbarkeit zeigt, weil diese plötzlich hereinbrechen, gegen den langsam einschleichenden und langsam wieder versehwindenden chemischen Reiz aber weniger empfindlich ist als in der Norm. Den Beweis kann man dadurch liefern, dass man auch den tactilen Reiz, von einem noch nicht wirksamen Minimum ausgehend, in allmäliger Steigerung applicirt. Setzt mản nämlich bei einem durch den Krampf geschwächten Präparate den Finger so leise auf, dáss noch kein Krampf erfolgt, drückt dann nach und nach etwas stärker, bis man die Reizschwelle sicher überschritten hat, und lässt ebenso langsam wieder nach, so erfolgt in der Regel kein Reflexkrampf. Dagegen erzeugt eine kurze Berührung, die hinter der mittleren Stärke des eben geschilderten Druckes etwas zurückbleibt, wieder einen Krampf.

Es ist also in Wirklichkeit auch hierin gar kein grundsätzlicher Unterschied zwischen chemischem und tactilem Reize vorhanden.

Am. Beobachten dieses Verhaltens gegen den chemischen Reiz wurde Schlick bei seinen Versuchen vor Allem durch seine zu hohen Strychningaben gehindert. So grosse Dosen, wie er sie verwendete, erzeugen nämlich sehr rasch einen Krampfanfall und damit die Abschwächung der Empfindlichkeit, während meine kleinen Dosen dem Beobachter eine längere Zeit (zwei bis zehn Minuten) zur Beobachtung der Reflexübererregbarkeit zur Verfügung stellen. Wie richtig dieser Einwand ist, geht aus Schlick's Bemerkung hervor (1. c. S. 179), dass dies Stadium der Kürze wegen sich schlecht beobachten liess und er allmälig in das spätere Stadium gerieth. Andererseits erholen sich auch die Thiere nach grösseren Strychnin- 
gaben von der Erschöpfung durch den Krampfanfall nicht mehr so gut wie nach kleineren Gaben. Vielmehr geht das Krampfstadium direct in das Stadium der Lähmung über, so dass die Wiederkehr der Empfindlichkeit gegen den chemischen Reiz nicht mehr beobachtet werden kann.

Ich will nun noch mehrere Einzelheiten der Technik anführen, in denen ich von Schlick abweiche; doch schreibe ich ihnen keinen wesentlichen Einfluss auf den Verlauf der Versuche zu. Zunächst muss ich erwähnen, dass ich stets Esculenten verwendet habe, weil diese im Allgemeinen zur Prüfung auf Reflexerregbarkeit sich besser eignen als Landfösche. Nach der Enthirnung liess ich die Frösche vor Austrocknen geschützt mindestens eine Stunde bei Zimmertemperatur stehen. In dieser Zeit haben sie sich bereits erholt und sind zum Experimentiren brauchbar. Ich hängte nun den Frosch anf und tauchte dann beide Hinterpfoten in ein weites Gefäss, das die Säureverdünnung enthielt, unter strengster Vermeidung des Anstossens an die Gefässwände.

Das Strychnin habe ich als Nitrat in Lösung $0,1: 1000$ verwendet; eine Pravaz'sche Spritze entspricht also 1/10 mg. In den folgenden Protokollen ist nur diese Concentration zu verstehen. Als Reizmittel habe ich Salzsäure angewendet.

\section{Versuch 65. Mittelgrosse Esculenta.}

12 h $30^{\prime}$ : 'Frosch wird aufgehängt, reagirt nicht auf leises Berühren der Zehen; auf leichten Druck zieht er an.

Salzsäure 1: 1000 .

12t 36': Nach 4" rasches Herausziehen beider Beine, heftiges Strampeln; rasch abgespült.

12 h 37': Dasselbe nach 1"'.

Salzsäure $1: 2000$.

$12{ }^{l} 40^{\prime}$ : Nach $5^{\prime \prime}$ dieselbe Reaction wie vorher, etwas schwächer.

12k 43': Dasselbe.

Salzsäure $1: 3000$.

12h 45': Nach 8" Herausziehen der Beine, Unruhe bis zum Abspülen.

12h $46^{\prime}$ : Dasselbe zwischen 8 und $10^{\prime \prime}$.

$12^{\text {h }}$ 47': Dasselbe zwischen 9 und 10".

$12^{\text {h }} 48^{\prime}$ : Dasselbe nach $10^{\prime \prime}$.

$12^{\text {h }} 50^{\prime}: 0,8 \mathrm{ccm}$ Strychninlösung.

$12^{\mathrm{h}} 55^{\prime}$ : Auf leise Berührung rasches Anziehen des Beines. Salzsäure: nach 12 " Heransaiehen, etwas lebhafter als vorher. 
12h 56': Nach 8" rasches Herausziehen, lebhaftes Strampeln.

Auf leise Berührung einer Hinterpfote rasches Anziehen beider Beine mehrmals binter einander.

$12^{\text {h }} 57^{\prime}$ : Nach $10^{\prime \prime}$ rasches Anziehen, dann mehrmaliges tactmässiges Ausstossen beider Beine; noch kein deutlicher Krampf.

12h 59': Auf Berührung zwei kurze Krampfstösse; bei Berühren der Wasseroberfläche Versuch anzuziehen, dann sofort Krampf von 15" Dauer.

1h $00^{\circ}$ : Bis $60^{\prime \prime}$ keine Reaction auf Säure. Auf Berührung Krampfstösse, ] $5^{\prime \prime}$ lang. Kurz darauf wirkt auch leises Berühren nicht mehr.

1h 02': Weder auf Säure noch auf einmaliges Berühren Reaction; bei der 3. Berührung kurzer Krampf.

$1 \mathrm{~h}$ 06': Krampfstoss im Augenblicke des Eintauchens; dasselbe geschieht beim Eintauchen in reines Wasser.

1h $08^{\prime}$ : Dasselbe.

1h 10': Dasselbe.

1h 11': Auf Säure nach 19" und nach $43^{\prime \prime}$ geringe Bewegungen des rechten Beines; kein Krampf.

1h $14^{\prime}$ : Salzsäure 1:2000:

Bei $9 "$ Versuch anzuziehen, nach 10" Krampfstoss.

$1^{\text {h }} 15^{\prime}$ : Nach 12" dasselbe.

1h $17^{\prime}$ : Salzsäure $1: 3000$ :

Bei $30 "$ und $" 24$ " dieselbe Reaction wie 1 h 14 '.

Durch Abspülen kurzer Krampf, beim Eintauchen und beim Herausnehmen.

1h 20': Berühren des Wassers, Säure und Berühren des Fingers sind wirkungslos.

1h 22': Bei 15" Versuch anzuziehen, gleich darauf Krampfstoss. Abspsülen: keine Reaction.

1' 24': Dasselbe nach $13^{\prime \prime}$.

1in $25^{\prime}$ : Dasselbe nach $12^{\prime \prime}$.

Versuch 66. Mittelgrosse Esculenta.

$4^{\text {h }} 00^{\prime}$ : Aufgehängt. Keine Reaction auf Berühren; Auziehen auf leichten Druck. $4^{\text {h }} 01^{\prime}: 0,25 \%$ ige Salzsäure verursacht fast momentan heftigste Reaction.

$4^{\text {h }} 05^{\prime}$ : $0,2 \%$ nach 5 " heftige Reaction.

$4^{\text {h }} 07^{\prime}: 0,1 \%$ nach $5^{\prime \prime}$ Herausziehen der Beine, lebhaftes Strampeln bis zum Abspülen.

4h $10^{\prime}: 0,05 \%$ nach 5 " Herausziehen, Unruhe bis zum Abspülen.

$4^{\text {h }} 11^{\prime}$ : Dasselbe nach $8^{\prime \prime}$.

4 h $12^{\prime}$ : Dasselbe.

$4^{h} 1^{\prime}$ : $1 / 24 \%$ nach $7^{\prime \prime}, 8$ " und $9^{\prime \prime}$ Herausziehen und Wiederfallenlassen der Beine; nach Entfernung aus der Säure keine Unruhe mehr.

$4^{\text {th }} 20^{\prime}: 0,6 \mathrm{ccm}$ Strychninlösung.

4h 25': Tactile Uebererregbarkeit.

Auf Säure $(1 / 24 \%)$ nach $11^{\prime \prime}$ rasches Herausziehen, heftiges Strampeln.

4h 28': Dasselbe nach 12".

$4^{\text {h }} 29^{\prime}:$ Dasselbe nach $10^{\prime \prime}$. 
$4^{\text {th }}$ 31': Nach 13" Anziehen, dann mehrmaliges heftiges Ausschlagen.

4h 32': Kurze spontane Krampfstösse.

$4^{\text {h }} 33^{\prime}$ : Nach 19" Herausziehen; gleich darauf heftiger Krampf von mehreren Secunden.

4h $35^{\prime}$ : Säure hat nach $50^{\prime \prime}$ noch nicht gewirkt; ebenso sind Berührungen wirkungslos.

$4^{\text {b }} 36^{\prime}$ : Bei Eintauchen in die Säure sofort (tactil) Krampfstoss; dasselbe nach $10^{\prime \prime}$ und $15^{\prime \prime}$.

4h 38': Bei Eintauchen Krampf, mit Unterbrechungen 1 Minute lang.

$4^{\mathrm{h}} 50^{\prime}$ : Bei mehreren Versuchen, den Frosch in die Säure zu tauchen, traten jedes Mal sofort (tactil) Krampfstösse auf.

$4^{\text {h }} 52^{\prime}$ : Beim Eintauchen Krampf von 2". Frosch verbleibt in der Säure, reagirt bis 50" nicht. Abspülen bleibt jetzt wirkungslos.

Versuch 69. Kleine Esculenta.

$12^{\text {h }} 14^{\prime}: 0,05 \%$ nach 6" Herausziehen, Unruhe bis zum Abspülen.

12h 19': Dasselbe nach $5^{\prime \prime}$.

12h 21': Dasselbe nach 6".

$12^{\text {h }} 26^{\prime}: 0,0375 \%$ nach 7 " und 8 " Herausziehen und Wiederfallenlassen der Beine.

12h 32': Dasselbe nach 8".

$12^{\text {h }} 35^{\prime}: 0,6 \mathrm{ccm}$ Strychninlösung.

$12^{\mathrm{h}} 44^{\prime}$ : Geringe tactile Uebererregbarkeit. 0,0375\% nach 11 " Herausziehen, Unruhe bis zum Abspülen.

12 h $45^{\prime}: 0,05 \%$ nach $8 "$, etwas stärker als vorher.

$12^{\mathrm{h}} 48^{\prime}: 0,0375 \%$ nach $8^{\prime \prime}$ dasselbe wie $12 \mathrm{~h} 44^{\prime}$.

$12^{\mathrm{h}} 51^{\prime}$ : $0,05 \%$ nach 8" Herausziehen, Strampeln bis zum Abspülen.

12b 53': 0,0375\% nach 5" Herausziehen, Unruhe bis zum Abspülen.

$12^{\mathrm{h}} 54^{\prime}$ : $0,0375 \%$ nach 9 ", etwas stärker als vorher.

12 h $56^{\prime}:$ Dasselbe nach $11^{\prime \prime}$.

$12^{\mathrm{h}} 57^{\prime}: 0,05 \%$ nach 8 " rasches Herausziehen, mehrmaliges Ausstossen.

$12 \mathrm{~h} 58^{\prime}: 0,0375 \%$ nach $5^{\prime \prime}$ rasches Herausziehen, Unruhe.

12h 59': $0,05 \%$ nach 8 " Herausziehen, Irradiation.

$1^{\text {th }} 00^{\prime}$ : Spontaner kurzer Krampfstoss.

1h 02': Beim Eintauchen kurzer Krampfstoss; nach $16^{\prime \prime}$ auf $0,05 \%$ geringes Anziehen, dann sofort Krampf.

Es bleibt nun noch ubbrig, bei der Prüfung auf allgemeine Empfindlichkeit das Verhalten des thermischen Reizes zu untersuchen. Da der thermische und der chemische Reiz in Bezug auf die Art ihres Einwirkens verwandt sind, so ist es von vornherein wahrscheinlich, dass thermische Reize ähnlich wie chemische wirken. Aus der kurzen früher citirten Bemerkung Schlick's über Versuche mit Wärmereizen einen Schluss auf diese Verwandtschaft zu ziehen, ist unmöglich. Denn auch hier hat Schlick weder die Reiz- 
stärke variirt noch die Energie der Reaction gemessen. Nicht einnıal die zwischen Reizung und Reaction abgelaufene Zeit wird mitgetheilt. Auch hat sich Schlick damit begnügt, die Empfindlichkeit gegen warmes Wasser zu prüfen.

Meine Versuche zeigen nun, dass ein enthirnter Frosch, der sich vorher in Wasser von ca. $20^{\circ} \mathrm{C}$. befunden hat, bei einer Zimmertemperatur von ebenfalls ca. $20^{\circ} \mathrm{C}$. vor der Vergiftung auf etwa $40^{\circ}$, nach der Vergiftung auf $37-38^{\circ}$ reagirt.

Durchgehends Temperatur des Frosches und des Zimmers = $20^{\circ} \mathrm{C}$.

Versuef 59. Mittelgrosse Esculenta.

$6^{\text {th }} 11-15^{\prime}: 36^{\circ}, 37^{\circ}$ und $38^{\circ}$ bleiben wirkungslos.

$6^{\text {h }} 16^{\prime}$ : $39^{\circ}$ nach $2^{\prime \prime}$ geringes Anziehen des rechten Beines.

6h 17': $40^{\circ}$ nach 2" mässig starkes Anziehen beider Beine, Verbleiben in dieser Haltung.

$6^{\text {h }} 19^{\prime}: 41^{0}$ sofort völliges Herausziehen beider Beine.

6 h 23': $0,8 \mathrm{ccm}$ Strychninlösung.

$6^{\text {h }} 31^{\prime}$ : Tactile Reflexübererregbarkeit. $38^{\circ}$ bei $2^{\prime \prime}$ und $4^{\prime \prime}$ geringes Anziehen.

6 ti $33^{\prime}$ : $37^{1 / 2^{0}}$ dasselbe.

6 h $35^{\prime}$ : $38^{\circ}$ nach $2^{\prime \prime}$ völliges Herausziehen beider Beine, anschliessend Krampfstoss.

Versuch 60. Mittelgrosse Esculenta.

$6^{\text {hi }} 48^{\prime}: 37^{\circ}$ keine Reaction.

6h 50': $38^{\circ}$ nach $2^{\prime \prime}$ mässig starkes Anziehen und Zurücksinkenlassen der Beine.

6h $52^{\prime}$ : $39^{\circ}$ etwas stärker als $38^{\circ}$, nach $2^{\prime \prime}$.

6 h $53^{\prime}: 40^{\circ}$ sofort völliges Herausziehen.

$6 \mathrm{~h} 55^{\prime}: 0,8 \mathrm{ccm}$ Strychninlösung.

$6 \mathrm{~h} 58^{\prime}:$ Tactile Uebererregharkeit.

$6^{\mathrm{h}} 59^{\prime}$ : $37^{\circ}$ sofort Herausziehen, mehrmals kräftiges Ausstossen, anschliessend

Krampf von mehreren Secunden.

Bei Reizversuchen mit kaltem Wasser war selbst mit Wasser von nur $1^{0}$ Wärme keine Reaction zu erzielen; nach der Vergiftung jedoch reagirten die Frösche auf $1-1^{1 / 2}{ }^{0}$ Wärme.

Versuch 63. Kleine Esculenta.

$12^{\mathrm{h}} 11-27^{\prime}: 4-1^{0}$ bleiben wirkungslos.

$12^{\text {h }} 29^{\circ}: 0,5 \mathrm{ccm}$ Strychninlösung.

12h 34': Keine Uebererregbarkeit. Nochmals $0,5 \mathrm{ccm}$ injicirt.

$12 \mathrm{~h} 40^{\prime}$ : Tactile Uebererregbarkeit.

$12^{\mathrm{h}} 41^{\prime}: 1^{\mathrm{o}}$ fast augenblicklich völliges Herausziehen der Beine.

$12^{\text {h }} 44^{\prime}: 1^{1 / 2^{0}}$ dasselbe; darauf mehrmaliges Ausstossen.

$12^{\text {h }} 45^{\prime}$ : Dasselbe; darauf Krampf. 
Versuch 64. Kleine Esculenta.

$12^{\mathrm{h}} 50^{\prime}: 2^{\circ}$ wirkungslos.

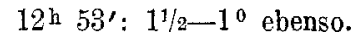

$12^{\mathrm{h}} 55^{\prime}: 1 \mathrm{ccm}$ Strychninlösung.

$1^{\text {h }} 00^{\prime}: 1^{0}$ sofort völliges Herausziehen.

$1^{\text {th }} 02^{\prime}: 1^{1 / 2^{0}}$ dasselbe, darauf Krampf.

Nach einem stärkeren Krampfanfalle zeigte sich auch für den thermischen Reiz eine Verminderung der Erregbarkeit; später trat die zuerst constatirte Steigerung der Empfindlichkeit wieder auf.

Ich komme nun zu den Versuchen betreffend die Veränderung der specifischen Reaction des Reflexfrosches auf chemische Reize.

Bei diesen Versuchen setzte ich den enthirnten Frosch auf eine befeuchtete Porzellanplatte. Er nimmt hier von selbst die Hockstellung ein; zieht man die Beine vom Körper ab, so werden sie sofort in die ursprüngliche Lage zurückgebracht. Auf leise Berührungen reagirt der hockende Frosch dureh Abwehrbewegungen oder auch durch schärferes Anziehen der Pfoten, auf Druck durch energische Abwehrbewegungen des ganzen Beines. Ich liess nun die Säure auf das Präparat einwirken, indem ich die Lösung mittelst einer Pipette vorsichtig auf Pfote und Unterschenkel eines Beines aufträufelte. Bei ausreichenden Concentrationen trat nun ein lebhaftes Wischen auf. Sobald die Reaction eingetreten war, ergriff ich den Frosch an einer Vorderpfote, spülte ihn in Leitungswasser $a b$ und setzte ihn auf eine andere befeuchtete Platte.

Nach Vergiftung mit Strychnin gelang es nun, zu zeigen, dass das Wischen des betreffenden Beines zunächst lebhafter wurde. Bei den folgenden Prüfungen wurde der Wischreflex undeutlich, weil er durch ausgesprochene Reflexirradiation gestört wurde: der Frosch streckte mehrmals hinter einander beide Beine mit grosser Schnelligkeit mehr oder weniger weit aus und zog sie wieder an. Schliesslich erfolgte auf der Höhe der Vergiftung als Abschluss einer solchen Reaction ein Krampfanfall von verschieden langer Dauer. Einige Male gelang es sogar, diese verstärkte Reaction hervorzurufen durch eine Concentration, die vor der Vergiftung unwirksam war. - Nach Ablauf des ersten Krampfes trat derselbe Gegensatz zwischen tactilem und chemischem Reize auf wie am hängenden Präparate: während zuweilen schon das Auffallen eines Tropfens als tactiler Reiz empfunden und mit einem Krampfe beantwortet wurde, blieb der anfangs wirksam chemische Reiz nunmehr völlig wirkungslos. Nach 
414 Hans Eckhardt: Chem. und therm. Reizung am strychninis. Frosche.

der Erholung stellte sich die typische Steigerung der Empfindlichkeit wieder her.

Die Zeit zwischen Beginn der Reizung und Reaction ist hier in Allgemeinen länger als bei den Versuchen am hängenden Präparate. Das liegt daran, dass die aufgeträufelte Flüssigkeit zum grossen Theile wieder abfliesst und man desshalb das Beträufeln einige Zeit fortsetzen muss, bis genug Säure aufgebracht ist. Beim hängenden Frosche wird also den sensiblen Nervenendigungen in der Zeiteinheit mehr Säure zugeführt. Dass nach der Vergiftung die Zeit zwischen Reizung und Reaction zuweilen wesentlich länger ist als vorher, hat seinen Grund darin, dass ich nach der Vergiftung vorsichtiger träufeln musste, um nicht durch das Auffallen des Tropfens eine (tactile) Reaction hervorzurufen. Hier darf also die Verlängerung der für uns bedeutungslosen "Latenzzeit" erst rẻcht nicht im Sinne von Schlick verwendet werden.

Versuch 31. Mittelgrosse Esculenta.

$7 \mathrm{~h} 05^{\prime}: 0,05 \%$ nach $40^{\prime \prime}$ geringe Reaction.

7 h $10^{\prime}: 0,075 \%$ nach 20 " mässig starkes Wischen.

7 h 25 : $0,05 \%$ nach $80 "$ geringes Wischen.

7 h $31^{\prime}: 0,075 \%$ nach $50 "$ dasselbe.

7 h $36^{\prime}: 0,1 \%$ nach $40 "$ lebhaftes Wischen.

7 h $40^{\prime}: 0,05 \%$ nach $60^{\prime \prime}$ geringe Reaction.

7 h $45^{\prime}: 0,075 \%$ nach 45 " dasselbe.

7 h $50^{\prime}: 0,1$ nach 40 " lebhaft.

$7 \mathrm{~h} 54^{\prime}: 1 \mathrm{ccm}$ Strychninlösung.

$8 \mathrm{~h} 00^{\prime}: 0,05 \%$ wie vorher.

$8 \mathrm{~h} 05^{\prime}$ : Tactile Uebererregbarkeit.

$0,05 \%$ nach $50 "$ lebhaftes Wischen, nach $100 "$ Krampf.

Versuch 53. Kleine Esculenta.

$7 \mathrm{~h} 30^{\prime}: 0,05 \%$ nach 40 " geringe Reaction.

7 h 32 ': $0,075 \%$ nach $20 "$ mässig lebhaft.

7 h 35 ': $0,1 \%$ nach 10 " sehr lebhaft.

7 h $36^{\prime}: 0,05 \%$ nach 30 " gering.

7h 38 ': $0,0375 \%$ wirkungslos.

7h $43^{\prime}$ : $0,8 \mathrm{ccm}$ Strychninlösung.

7 h 47': Deutliche Ueberregbarkeit.

$0,05 \%$ nach $2 "$ sehr heftiges Wischen.

7h 48': 0,0375\% nach 30" lebhafte Reaction-Irradiation-Krampf.

Für den typischen Wischreflex gelten also dieselben Gesichtspunkte, wie wir sie oben für die Prüfung der allgemeinen Empfindlichkeit chemisehen Reizen gegentuber schon durchgesprochen haben. 